\title{
Sialometaplasia Necrotizante: Revisión de la Literatura a Propósito de un Caso
}

\author{
Necroting Sialometaplasia: Review of the Literature on a Case
}

Edgard Riquelme M. ${ }^{1}$ \& Rodrigo Badilla M. ${ }^{1,2,3}$

RIQUELME, M. E. \& BADILLA, M. R. Sialometaplasia Necrotizante: Revisión de la Literatura a Propósito de un Caso. Int. J. Odontostomat., 12(2):142-146, 2018.

RESUMEN: La sialometaplasia necrotizante $(\mathrm{SN})$ es una rara enfermedad benigna, inflamatoria, autolimitante, que afecta más frecuentemente a las glándulas salivales menores y que comúnmente se asocia a las ubicadas en la porción más posterior del paladar duro. Su etiología no esta clara, la mayoría de los autores sugieren que una lesión química, física o biológica de los vasos sanguíneos produciría cambios isquémicos, que provocarían infarto del tejido glandular con necrosis, inflamación e intento de reparación. Clínicamente puede presentarse como una úlcera de márgenes irregulares, ligeramente elevados y lecho necrótico, mientras que histopatológicamente se caracteriza por presentar metaplasia escamosa de conductos y acinos e hiperplasia pseudoepiteliomatosa del epitelio mucoso. La similitud de sus características clínicas e histopatológicas con algunas lesiones glandulares malignas de la cavidad oral, puede resultar en tratamientos innecesarios, considerando que la SN se trata de una patología autoresolutiva, por lo que es fundamental realizar un correcto diagnóstico clínico e histopatológico para evitar tratamientos quirúrgicos mutilantes o innecesarios. En el presente trabajo se presenta un caso de una mujer joven, con diagnóstico de SN, con sus características clínicas, histopatológicas y la evolución de la lesión.

PALABRAS CLAVE: sialometaplasia necrotizante, glándulas salivales, úlcera oral.

\section{INTRODUCCIÓN}

La sialometaplasia necrotizante (SN) corresponde a un proceso inflamatorio, benigno y autolimitante. Se presenta principalmente en sitios con glándulas salivales menores, habitualmente en el paladar duro o en el límite entre el paladar duro y blando. Sin embargo, es posible observarla en diferentes sitios de la cavidad oral (Papanayotou et al., 1980), en glándulas salivales mayores (Rossie et al., 1986), en mucosa sinusal (Hovinga \& De Jager, 1977) y en la laringe (Grillon \& Lally, 1981).

La etiología de esta afección no es clara, la mayoría de los autores sugieren que una agresión física, química o biológica sobre los vasos sanguíneos produciría una lesión isquémica, la cual provocaría infarto del tejido glandular y su posterior necrosis (Suckiel et al., 1978), inflamación e intento de reparación induciendo metaplasia, cambios en ductos y pos- terior cicatrización (Chaudhry et al., 1985). Traumatismos locales directos, como los producidos por intubaciones, aplicación de anestesia local asociada a vasoconstrictores (Shigematsu et al., 1996), procedimientos quirúrgicos, uso de prótesis desajustadas, vómitos violentos o provocados, como los descritos en pacientes con bulimia (Schöning et al., 1998), radioterapia, uso de tabaco (Fechner, 1977) y cocaína entre otros (Imbery \& Edwards, 1996) serían factores involucrados en esta alteración vascular. Algunos autores encuentran relación con patologías tales como diabetes o alcoholismo crónico (Murphy et al., 1977).

Clínicamente la SN puede presentarse como una úlcera con aspecto crateriforme, de márgenes indurados y bien delimitados, ubicada comúnmente en paladar, pudiendo ser bilateral (Raugi \& Kessler, 1979; Samit et al., 1979); o bien como un aumento de volu-

\footnotetext{
${ }^{1}$ Servicio de Urgencia Bucomaxilofacial, Hospital de Urgencia Asistencia Pública (HUAP) Dr. Alejandro del Río, Santiago, Chile

${ }^{2}$ Servicio de Cirugía Maxilofacial, Hospital Félix Bulnes Cerda, Santiago, Chile

${ }^{3}$ Departamento de Cirugía y Traumatología Maxilofacial, Facultad de Odontología, Universidad Andrés Bello, Santiago, Chile.
} 
men de consistencia fluctuante (Santis et al., 1982), doloroso o asintomático y con un tamaño entre 0,7 5,0 centímetros de diámetro, aproximadamente (Joshi et al., 2014). Las características histopatológicas incluyen metaplasia escamosa de conductos y acinos, necrosis por coagulación de algunos lóbulos (Favia et al., 1996), presencia de tejido de granulación con infiltrado inflamatorio inespecífico e hiperplasia pseudoepiteliomatosa del epitelio mucoso superficial (Williams et al., 1979).

Esta enfermedad es relativamente inusual, con una prevalencia bajo el $1 \%$ del total de las biopsias (Joshi et al.). La importancia de esta lesión es que por su aspecto clínico y algunas de sus características histológicas, fácilmente se confunde con neoplasias malignas orales como el Carcinoma Adenoideo Quístico, el Carcinoma Mucoepidermoide o el Carcinoma de Células Escamosas (Fechner; Suckiel et al.). Por lo tanto es fundamental un adecuado diagnóstico clínico e histopatológico para evitar tratamientos quirúrgicos mutilantes e innecesarios.

\section{CASO CLÍNICO}

Paciente sexo femenino, de 19 años de edad, sin antecedentes medico-quirúrgicos de relevancia. Fumadora de 3 cigarrillos diarios hace 6 años. Concurre
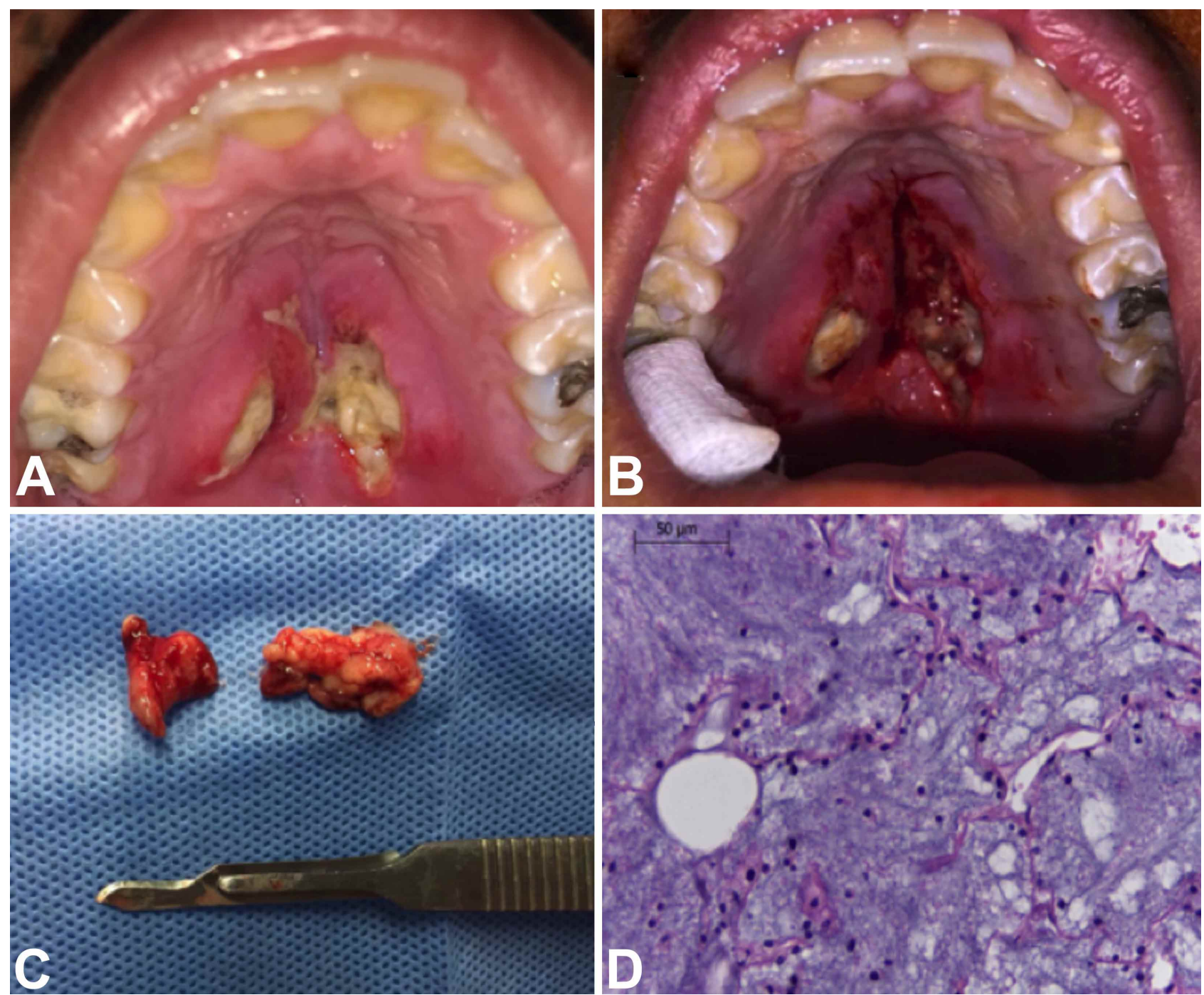

Fig. 1. A) Evolución de la lesión a los 10 días, B) Biopsia incisional de la lesión, C) Muestra de tejido biopsiado y D) Microfotografía de corte histológico tinción hematoxilina-eosina x50. Se observan algunos ductos dilatados en corion entre infiltrado mononuclear, también varios lobulillos glandulares necróticos, con persistencia de los límites de los acinos, con citoplasmas basófilos y núcleos hipercromáticos. 
al Servicio de Urgencia Bucomaxilofacial HUAP por presentar lesiones ulcero necróticas en región de paladar duro y blando, en toda su extensión, de 10 días de evolución. Relata que en un inicio las lesiones se presentaron como aumentos de volumen fluctuantes, de aparición brusca, dolorosas (EVA 5) y cuya sintomatología fue disminuyendo paulatinamente. Al tercer día acude a consulta privada donde se indica tratamiento antibiótico de cloxacilina 500 miligramos. cada 8 horas por 7 días; sin mejorías de las lesiones. Cabe destacar que la paciente niega otro tipo de hábitos, como el consumo de cocaína, no relata atenciones odontológicas recientes y tampoco historia de traumatismos de la mucosa oral que pudiesen estar asociados a la aparición de las lesiones.

Clínicamente se observan lesiones ulcerosas bilaterales, que comprometen paladar duro y blando, de lecho necrótico, no doloras a la palpación, cuyos márgenes son definidos, solevantados y eritematosos (Fig.
1A). Se indica biopsia incisional de la lesión (Fig. 1B,C), exámenes de laboratorio (hemograma completo, perfil de coagulación y bioquímico) y tomografía axial computada (TAC) maxilofacial contrastada. Los exámenes de laboratorio arrojaron resultados normales y la imageneología no evidenció compromiso óseo maxilar (Fig. 2). En el informe histopatológico se observó varios fragmentos de mucosa con escaso epitelio de revestimiento, con algunos ductos dilatados en corion entre infiltrado mononuclear y otros fragmentos de tejido necrótico con algunas colonias bacterianas, también varios lobulillos glandulares necróticos con persistencia de los límites de los acinos, con citoplasmas basófilos y núcleos hipercromáticos (Fig. 1D), lo que indica una lesión de origen posiblemente traumático. El diagnóstico fue de SN. A los 14 días de realizada la biopsia, el lecho estaba cubierto por tejido de granulación cicatrizando por segunda intención (Fig. 3A). La lesión remitió espontáneamente a las 7 semanas posteriores a la biopsia (Fig. 3B).
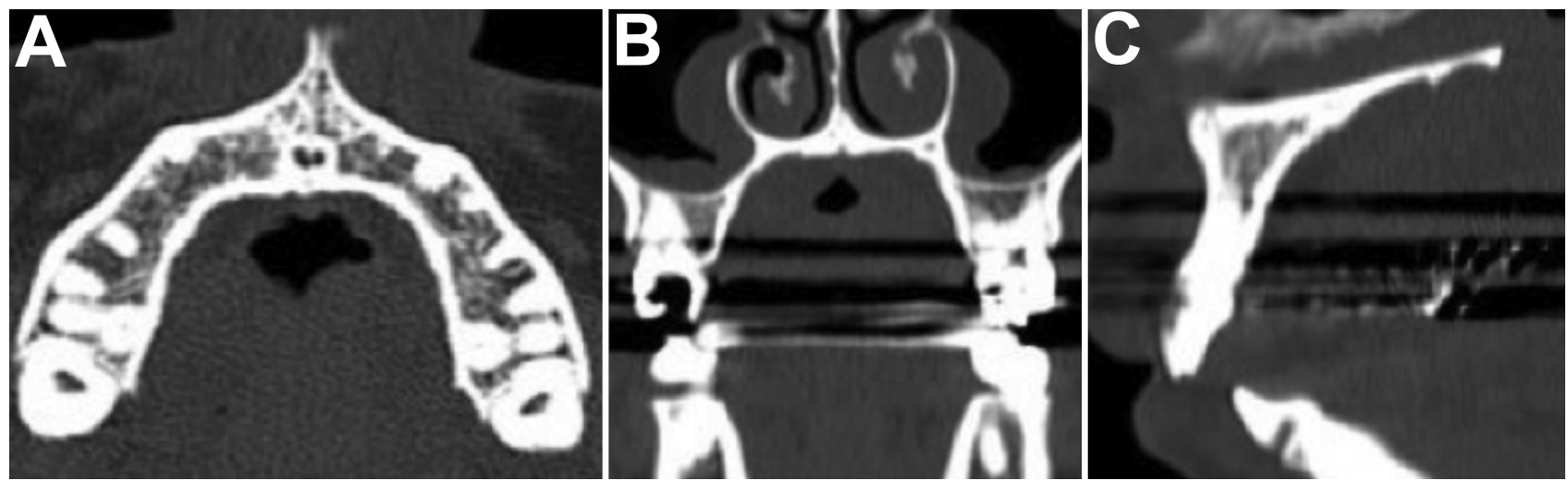

Fig. 2. Imágenes de tomografía axial computada (TAC) donde se observa indemnidad de las corticales óseas en el plano axial $(A)$, coronal $(B)$ y sagital $(C)$.
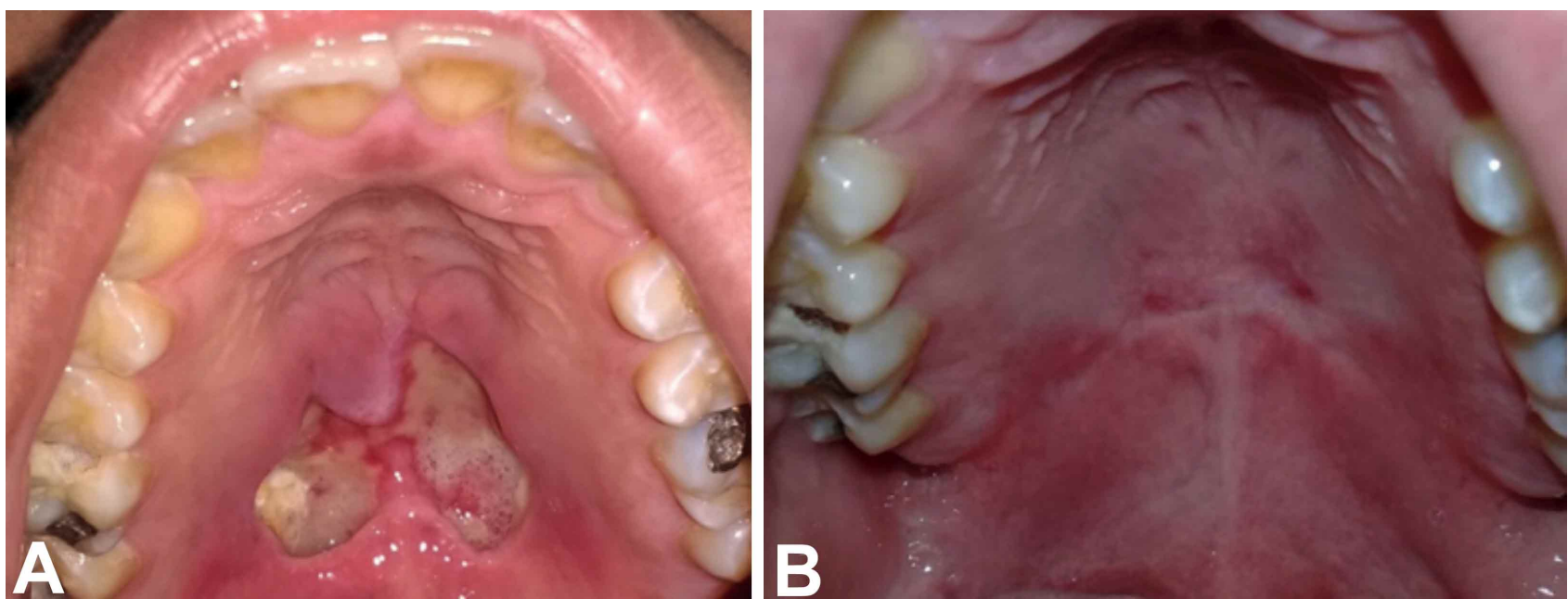

Fig. 3. A) Evolución de la lesión a los 14 días posteriores a la biopsia y B) A las 7 semanas. Donde se aprecia mucosa palatina indemne. 


\section{DISCUSIÓN}

La SN fue descrita por primera vez en 1973 por Abrams et al. (1973) como una lesión inflamatoria necrotizante de las glándulas salivales menores del paladar duro. Se trata de una afección relativamente rara, con una mayor prevalencia en mujeres de mediana edad (Rye et al., 1980), cuya presentación clínica más común es la ulcerativa (Birkholz et al., 1979) y que se presenta generalmente de forma unilateral, aunque existen reportes sobre SN bilaterales y en la línea media. En el caso antes descrito, las lesiones ulcerativas se presentaron en una mujer joven, cuya localización comprometió paladar duro y blando, siendo bilaterales y que abarcaban la línea media palatina.

Si bien, su etiopatogenia es aún desconocida, la mayoría de los autores coinciden con la teoría de la isquemia glandular y la relación de esta lesión con antecedentes de trauma o irritantes locales tales como el tabaco, consumo de cocaína, ácido gástrico en cuadros de reflujo gastroesofágico o bulimia (Imai \& Michizawa, 2013), alcohol, radiación, infecciones de la vía aérea superior, procedimientos quirúrgicos e infiltración de anestesia local en la zona (Carlson, 2009). Este último punto de describe con frecuencia en la literatura, sobre todo asociado al uso de vasoconstrictores en mucosa palatina. En el caso descrito, la lesión puede estar asociada principalmente al uso prolongado de irritantes locales, específicamente el consumo de tabaco, ya que se descartaron otros factores locales de trauma y factores sistémicos concomitantes.

La relación entre la $\mathrm{SN}$ y los antecedentes de trauma o agentes irritantes locales, es un factor importante al momento de diagnosticar la enfermedad, ya que las similitudes clínicas e histológicas con lesiones malignas de la cavidad oral como el Carcinoma Mucoepidermoide, Carcinoma Adenoide Quístico y el Carcinoma de Células Escamosas (Fechner; Suckiel et al.), pueden llevar a planes de tratamientos invasivos, mutilantes e innecesarios.

Se han descrito 3 criterios histológicos para distinguir la SN de lesiones glandulares malignas: preservación de la morfología lobular general, islas de células escamosas sin signos de malignidad evidente en su citología y que no se observen residuos ductales en dichas islas (Brannon et al., 1991).

Por otra parte, Anneroth \& Hansen (1982) pro- pusieron 5 etapas histológicas en el desarrollo de la SN: infarto, secuestro, ulceración, reparación y curación. Según este esquema, una etapa de infarto más prolongada, seguida del secuestro de acinos necróticos, resultaría en la formación de una úlcera. Mientras que una etapa de infarto más limitada no se produciría los secuestros ni la úlcera. Esta podría ser la razón de las 2 formas de presentación clínica de la SN (Anneroth \& Hansen).

Habitualmente la SN no requiere tratamiento, y las lesiones ulceradas cicatrizan espontáneamente por segunda intención entre 4-10 semanas (Joshi et al.). Se debe destacar que al tomar la biopsia, esta debe abarcar tejido sano, para evitar enviar solo zonas necróticas en el estudio histopatológico (Balaji \& Balaji, 2015). En el caso descrito, la resolución del cuadro clínico se limitó a los tiempos descritos en la literatura.

\section{CONCLUSIÓN}

La SN es una patología rara, benigna y autoresolutiva que afecta las glándulas salivales menores, que puede confundirse frecuentemente con lesiones malignas de la cavidad oral y por ende, puede desencadenar tratamientos mutilantes e innecesarios. Para evitar esto, es fundamental considerar la historia y hallazgos clínicos tras estas lesiones. Además, es de suma importancia la colaboración constante de especialistas en cirugía y patología oral para el diagnóstico clínico e histopatológico certero de la lesión, dado el amplio número de patologías que pueden afectar a las glándulas salivales.

\section{AGRADECIMIENTOS}

Al Dr. Benjamín Martínez Rondanelli, por su colaboración con el estudio histopatológico del caso descrito.

RIQUELME, M. E. \& BADILLA, M. R. Necroting sialometaplasia: Review of the Literature on a Case. Int. J. Odontostomat., 12(2):142-146, 2018.

ABSTRACT: The Necrotizing Sialometaplazia (NS) it's a rare self-limiting, inflammatory, benign disease, that most frequently affects the minor salivary glands and it is commonly associated to the glands located at the most posterior portion 
of the hard palate. Its etiology is not clear. Most authors suggest that a chemical, physical or biological lesion of the blood vessels would produce ischemic changes, which lead to infarction of muscle tissue with necrosis, inflammation and attempts to repair. Clinically it can present as a slightly elevated ulcer with irregular edges and necrotic bed, while histopathologically it is characterized for present squamous metaplasia of ducts and acini and pseudoepitheliomatous hyperplasia of mucosal epithelium. The similarity of its clinical and histopathological characteristics with some malignant glandular lesions of the oral cavity, can result in unnecessary treatments, considering that NS is a self-sustaining pathology, it is therefore essential to perform a correct clinical and histopathological diagnosis to avoid mutilating or unnecessary surgical treatments. In the present work we present the case of a young woman, with diagnosis of NS, with its clinical and histopathological characteristics and the evolution of the lesion.

KEY WORDS: necrotizing sialometaplasia, salivary glands, oral ulcer.

\section{REFERENCIAS BIBLIOGRÁFICAS}

Abrams, A. M.; Melrose, R. J. \& Howell, F. V. Necrotizing sialometaplasia. A disease simulating malignancy. Cancer, 32(1):130-5, 1973.

Anneroth, G. \& Hansen, L. S. Necrotizing sialometaplasia. The relationship of its pathogenesis to its clinical characteristics. Int. J. Oral Surg., 11(5):283-91, 1982.

Balaji, S. M. \& Balaji, P. Surgical management of necrotizing sialometaplasia of palate. Indian J. Dent. Res., 26(5):550-5, 2015.

Birkholz, H.; Minton, G. A. \& Yuen, Y. L. Necrotizing sialometaplasia: review of the literature and report of nonulcerative case. J. Oral Surg., 37(8):588-92, 1979.

Brannon, R. B.; Fowler, C. B. \& Hartman, K. S. Necrotizing sialometaplasia. A clinicopathologic study of sixty-nine cases and review of the literature. Oral Surg. Oral Med. Oral Pathol., 72(3):317-25, 1991.

Carlson, D. L. Necrotizing sialometaplasia: a practical approach to the diagnosis. Arch. Pathol. Lab. Med., 133(5):692-8, 2009.

Chaudhry, A. P.; Yamane, G. M.; Salman, L.; Salman, S.; Saxon, M. \& Pierri, L. K. Necrotizing sialometaplasia of palatal minor salivary glands: a report on 2 cases. J. Oral Med., 40(1):2-6, 1985.

Favia, G.; Pratelli, D. \& Maiorano, E. La sialometaplasia necrotizzante. Analisi clinico-patologica di 6 casi. Minerva Stomatol., 45:121-7, 1996.

Fechner, R. E. Necrotizing sialometaplasia: a source of confusion with carcinoma of the palate. Am. J. Clin. Pathol., 67(4):315-7, 1977.

Grillon, G. L. \& Lally, E. T. Necrotizing sialometaplasia: literature review and presentation of five cases. J. Oral Surg., 39(10):74753,1981

Hovinga, J. \& De Jager, H. A patient with necrotizing sialometaplasia. Int. J. Oral Maxillofac. Surg., 6(5):280-2, 1977.

Imai, T. \& Michizawa, M. Necrotizing sialometaplasia in a patient with an eating disorder: palatal ulcer accompanied by dental erosion due to binge-purging. J. Oral Maxillofac. Surg., 71(5):87985,2013
Imbery, T. A. \& Edwards, P. A. Necrotizing sialometaplasia: literature review and case reports. J. Am. Dent. Assoc., 127(7):1087-92, 1996.

Joshi, S. A.; Halli, R.; Koranne, V. \& Singh, S. Necrotizing sialometaplasia: A diagnostic dilemma! J. Oral Maxillofac. Pathol., 18(3):420-2, 2014.

Murphy, J.; Giunta, J.; Meyer, I. \& Robinson, K. Necrotizing sialometaplasia. Oral Surg. Oral Med. Oral Pathol., 44:419-24, 1977.

Papanayotou, P. H.; Kayavis, J. G.; Epivatianos, A. A. \& Trigonidis, G. Necrotizing sialometaplasia of the cheek: report of case and review of literature. J. Oral Surg., 38(7):538-40, 1980.

Raugi, G. J. \& Kessler, S. Necrotizing sialometaplasia. A condition simulating malignancy. Arch. Dermatol., 115(3):329-31, 1979.

Rossie, K. M.; Allen, C. M. \& Burns, R. A. Necrotizing sialometaplasia: a case with metachronous lesions. J. Oral Maxillofac. Surg., 44(12):1006-8, 1986.

Rye, L. A.; Calhoun, N. R. \& Redman, R. S. Necrotizing sialometaplasia in a patient with Buerger's disease and Raynaud's phenomenon. Oral Surg. Oral Med. Oral Pathol., 49(3):233-6, 1980.

Samit, A. M.; Mashberg, A. \& Greene, G. W. Jr. Necrotizing sialometaplasia. J. Oral Surg., 37(5):353-6, 1979.

Santis, H. R.; Kabani, S. P.; Roderiques, A. \& Driscoll, J. M. Necrotizing sialometaplasia: an early, nonulcerative presentation. Oral Surg. Oral Med. Oral Pathol., 53(4):387-90, 1982.

Schöning, H.; Emshoff, R. \& Kreczy, A. Necrotizing sialometaplasia in two patients with bulimia and chronic vomiting. Int. J. Oral Maxillofac. Surg., 27(6):463-5, 1998.

Shigematsu, H.; Shigematsu, Y.; Noguchi, Y. \& Fujita, K. Experimental study on necrotizing sialometaplasia of the palate in rats. Role of local anesthetic injections. Int. J. Oral Maxillofac. Surg., 25(3):239-41, 1996.

Suckiel, J. M.; Davis, W. H.; Patakas, B. M. \& Kaminishi, R. M. Early and late manifestations of necrotizing sialometaplasia. J. Oral Surg., 36(11):902-5, 1978.

Williams, R. F. Necrotizing sialometaplasia after bronchoscopy. J. Oral Surg., 37(11):816-8, 1979.

Dirección para correspondencia:

Edgard Riquelme M

Servicio de Urgencia Bucomaxilofacial

Hospital de Urgencia Asistencia Pública (HUAP)

Dr. Alejandro del Río

Santiago

CHILE

Email: edgard.riquelme.medel@gmail.com

Recibido : 18-12-2017

Aceptado: 05-02-2018 